\title{
Laboratory diagnosis and follow-up of Romanian Gaucher disease patients
}

\author{
Cristina Drugan ${ }^{1}$, Tudor Drugan ${ }^{2 *}$, Catherine Caillaud ${ }^{3}$, Paula Grigorescu-Sido ${ }^{4}$, \\ Tiberiu Nistor ${ }^{1}$, Alexandra M. Crăciun ${ }^{1}$
}

1. Department of Medical Biochemistry, „Iuliu Haţieganu” University of Medicine and Pharmacy, Cluj-Napoca, Romania

2. Department of Medical Informatics and Biostatistics, „Iuliu Haţieganu” University of Medicine and Pharmacy, Cluj-Napoca, Romania

3. Laboratoire de Biochimie, Métabolomique et Protéomique, Hôpital Necker-Enfants

Malades, University Paris Descartes, France

4. Department of Paediatrics I, ,,Iuliu Haţieganu” University of Medicine and Pharmacy, Cluj-Napoca, Romania

\begin{abstract}
Background: Gaucher disease (GD) is caused by a recessively inherited deficiency of glucocerebrosidase which is encoded by the GBA gene in which nearly 450 mutations have been described. However, only a few genotype-phenotype correlations have been clearly established. The aim of this study was to investigate molecular features of GD in Romanian patients and to evaluate their impact on treatment response.

Material and methods: 69 patients, diagnosed between 1997 and 2014 at our national referral laboratory, were included in this study. Frequent point mutations (N370S, L444P, 84GG, R463C) were detected by amplification and restriction enzyme digestion. Recombinant alleles (recTL, recNciI, recA456P) were screened by DNA sequencing. Plasma chitotriosidase served as a biomarker of disease severity throughout the follow-up period.

Results: 66 patients had the non-neuronopathic (type 1) form of GD and 3 had the chronic neuronopathic (type 3) phenotype. We identified 79\% of the mutant alleles, among which the most frequent mutations were N370S (54\%) and L444P (18\%). We found a statistically significant $(p<0.001)$ and moderate to good correlation between the total therapeutic dose and the residual chitotriosidase activity $(R=0.621)$. After two years of treatment, we noticed statistically significant variations in chitotriosidase activity corresponding to the most frequent genotypes (N370S) unknown allele, N370S/L444P, N370S/N370S and N370S/R463Q).

Conclusions: Allele distribution displayed specific features in Romanian GD patients, such as the high prevalence of the N370S allele. Chitotriosidase activity measurement allowed the investigation of genotype influence on treatment outcome.

Keywords: Gaucher disease, genotype-phenotype correlations, chitotriosidase, enzyme replacement therapy, Romanian population

Received: $26^{\text {th }}$ February 2017; Accepted: 3rd May 2017; Published: $24^{\text {th }}$ May 2017
\end{abstract}

*Corresponding author: Tudor Drugan, Department of Medical Informatics and Biostatistics, „Iuliu Haţieganu” University of Medicine and Pharmacy, Cluj-Napoca, Romania, e-mail: tdrugan@umfcluj.ro 


\section{Introduction}

Gaucher disease (GD), the most prevalent sphingolipid storage disorder, results from a recessively inherited deficiency of glucocerebrosidase (EC 3.2.1.45). The gene encoding this lysosomal enzyme $(G B A)$ is located on chromosome $1 \mathrm{q} 21$, in close proximity to a highly homologous pseudogene (1). To date, around 450 mutations have been described in the $G B A$ gene, including substitutions, insertions, deletions and recombinant alleles, resulting from recombination events between the functional gene and its pseudogene (2).

A progressive build-up of the undegraded substrate, glucosylceramide, in the lysosomes of macrophages, triggers a cascade of pathogenic events: the activation and conversion of macrophages into the characteristic "Gaucher cells", their accumulation in various tissues and the synthesis of pro-inflammatory cytokines by these activated macrophages, leading to a persistent state of subclinical inflammation (3).

The clinical picture is dominated by hepatosplenomegaly, anaemia, thrombocytopenia and varying degrees of skeletal and neurological involvement. Type 1 GD (the non-neuronopathic variant) is defined by the absence of primary neurological involvement, while a progressive neurodegenerative course is the hallmark of the disease in types 2 (the acute neuronopathic form) and 3 (the chronic neuronopathic subtype) (4). Current therapeutic options rely on enzyme replacement therapy (ERT) with macrophage-targeted glucocerebrosidase and substrate reduction therapy (SRT), monitored by several biomarkers, such as chemokines or enzymes produced by Gaucher cells $(5,6)$. Among these, circulating chitotriosidase is widely regarded as the primary biomarker reflecting global storage cell burden.

Although a few $G B A$ mutations account for most of the disease-related genotypes in the Jewish population (N370S, L444P, 84insG and IVS2 $+1 \mathrm{G}>A$ ), a broad spectrum of genetic vari- ability has been described in different populations (7-9). However, only a few genotype-phenotype correlations have been clearly established, namely the association of the N370S allele with type $1 \mathrm{GD}$ and the high prevalence of the L444P allele in neuronopathic disease (10).

In a preliminary study, we have already reported the mutation spectrum of GD in our country, but it concerned only a small number of patients referred to our laboratory during its first 5 years of activity (11). In the meantime, our data increased progressively. Herewith, we intend to further explore the molecular features of GD in patients originating from all over our country and to evaluate the impact of various genotypes on treatment response, assessed by chitotriosidase monitoring.

\section{Material and methods}

\section{Patients}

A total of 69 patients, diagnosed with GD between 1997 and 2014 at our national referral laboratory were included in this study. The diagnosis was based on clinical criteria and confirmed by demonstration of deficient glucocerebrosidase activity (less than $20 \%$ of the reference values) in peripheral blood leukocytes (12). Patient information (age at diagnosis and at treatment initiation, ERT dose and biological findings) were recorded at diagnosis and during subsequent evaluations, scheduled yearly before ERT and twice a year afterwards.

This study was approved by the Ethics Committee of our University and an informed written consent was signed by all patients or their legal representatives. Patient samples were collected during routine diagnostic work-up and subsequent follow-up evaluations.

\section{Mutation analysis}

The presence of frequent GBA point mutations was investigated by polymerase chain 
reaction (PCR) amplification and restriction enzyme digestion. The alleles N370S, L444P, $84 \mathrm{GG}$ and R463C (according to the traditional mutation nomenclature still commonly used) were screened according to the previously described methods (13-15). The recombinant alleles recTL (mutations D409H, L444P, A456P, V460V), recNciI (mutations L444P, A456P, $\mathrm{V} 460 \mathrm{~V}$ ) and recA456P (mutations L444P, A456P) were detected by sequencing of the amplified fragments harbouring the L444P substitution, on an automated ABI 373A DNA sequencer with the Big Dye Terminator Ampli Taq FS kit (ABI), according to the manufacturer's recommendations.

\section{Chitotriosidase activity assay}

Plasma chitotriosidase activity was determined using an artificial substrate (4-methyl-umbelliferyl- $\beta$-D- $N, N$ ', $N$ '”-triacetylchitotrioside), according to the previously described enzymatic assay (16). Circulating chitotriosidase was used as a marker of disease burden at diagnosis and throughout the follow-up period for each patient, except those in which a complete deficiency, reported to occur in about $6 \%$ of the population including patients with GD (17), had been detected.

\section{Statistical analysis}

Microsoft Excel was used for data collection and analysis. Statistical analysis was performed with the IBM SPSS software: normality was assessed with Kolmogorov-Smirnov and Shapiro-Wilk tests, frequency distribution was verified with Chi-square and Fisher-exact tests and quantitative variables were compared with Kruskal-Wallis and Mann-Whitney U tests, assuming a 0.05 significance level. Logarithmic regression analysis and regression significance testing were performed in SigmaPlot using a dynamic fit.

\section{Results \\ Patient characteristics}

Among the 69 GD patients diagnosed between 1997 and 2014, 39 were females (57\%) and 30 were males (43\%). All of them were registered as Caucasians, of non-Jewish ethnicity. Patients originated from the entire territory of our country. 66 patients were considered to have the non-neuronopathic (type 1) form, based on the absence of primary neurological involvement. Only 3 patients had the chronic neuronopathic (type 3) phenotype. Type 2, the acute neuronopathic form, was not identified in our patients. During our study, four patients died (three with type 1 and one with type 3 ) and other four were lost to follow-up. At diagnosis, the mean age was $28.74 \pm 15.81$ years (range $2-70$ ).

\section{Distribution of mutant alleles and geno- types}

After excluding the affected siblings of each index case, we studied the distribution of mutations in the 58 unrelated GD patients, irrespective of the disease type. Screening for the most frequent mutations in the $G B A$ gene allowed the characterisation of $79.31 \%$ of the mutant alleles (92 alleles), while 20.69\% (24 alleles) remained unknown (figure 1). Mutations $84 \mathrm{GG}$ and recTL have not been found in our patients. The most frequent mutations were N370S, found in 63 alleles and accounting for more than half of the disease-related alleles, and L444P, occurring in 21 alleles. The other mutations were recNciI (3 alleles), R463Q (3 alleles), recA456P (1 allele) and $\mathrm{R} 463 \mathrm{C}$ (1 allele).

Next, we compared the prevalence of mutant alleles in 56 unrelated type 1 GD patients with those reported in a large number of Ashkenazi Jewish and non-Jewish patients (18). These results are illustrated as stacked columns in figure 2. Allele distribution displayed considerable differences compared to both non-Jewish and Jewish populations. Similar frequencies were found 


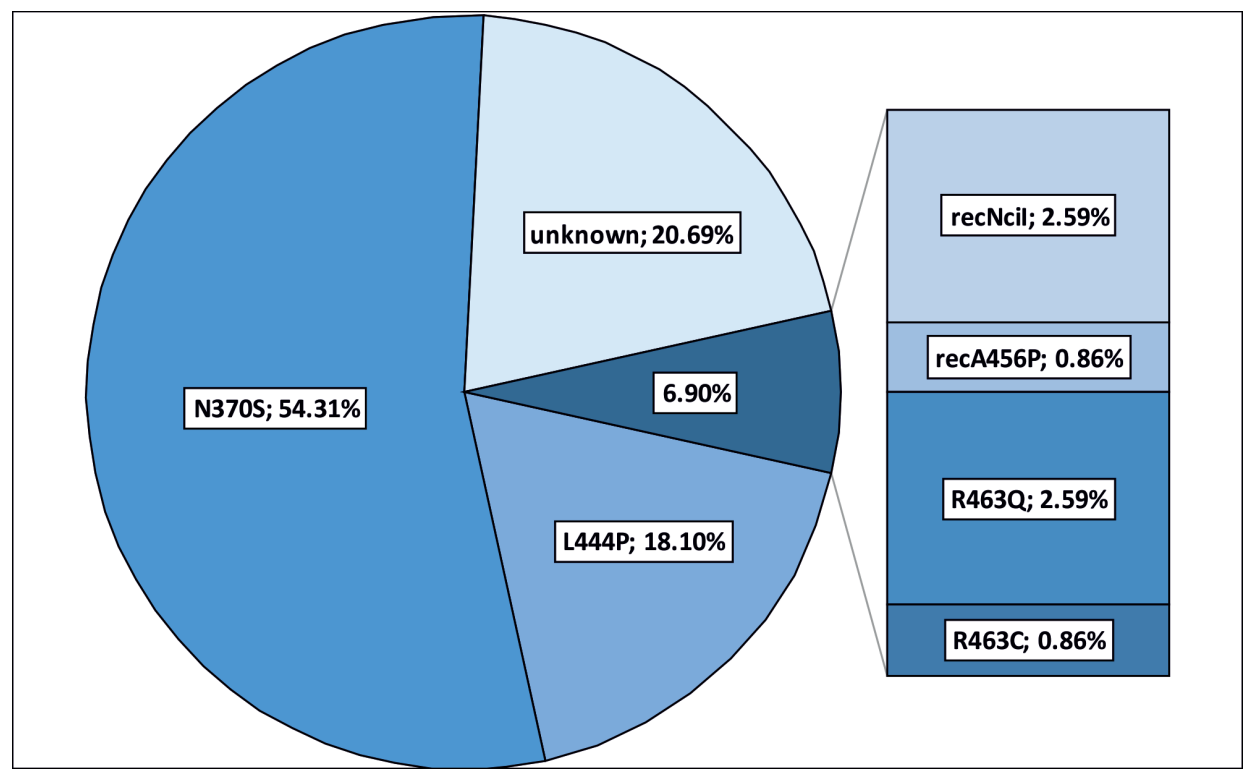

Figure 1. Allele distribution in 58 unrelated (types 1 and 3) Romanian GD patients

only for the L444P allele in our patients and in non-Jewish Caucasians, while the N370S and the sporadic, unidentified, mutations had an intermediate prevalence, between non-Jewish and Jewish populations. Another difference concerns the R463C allele, whose prevalence was lower in our patients than in non-Jewish populations. Overall, the differences between allele frequencies in our patient sample and the reference ethnic groups were highly significant, with $\mathrm{p}\left(\chi^{2}\right.$ $>34.847)<0.001$ and $\mathrm{p}\left(\chi^{2}>18.982\right)<0.001$ for the comparison with Ashkenazi Jewish and non-Jewish populations, respectively.

Most genotypes of our patients had at least one N370S allele and, as expected, corresponded to type 1 GD (figure 3 ). Among the 56 unrelated type 1 patients, the most prevalent genotypes were N370S/unknown allele (21 patients), N370S/L444P (18 patients) and N370S/N370S ( 8 patients). The genotypes of the 2 unrelated patients with type $3 \mathrm{GD}$ were L444P/L444P and L444P/unknown allele, respectively.

Again, the comparison of genotype frequencies between our patients with type 1 GD and a large number of Jewish (19) and non-Jewish patients (18) revealed several differences, such as, in our patients, the lower prevalence of genotypes N370S/recNciI and unknown/unknown, or the high frequency of N370S homozygotes, although this genotype is less frequent in non-Jewish patients. Compound heterozygotes with the genotype N370S/unknown allele were found with comparable frequencies among our patients and non-Jewish Caucasians. The differences between genotype frequencies in our population and the reference ethnic groups were statistically significant, with $\mathrm{p}\left(\chi^{2}>54.770\right)<0.001$ and $\mathrm{p}$ $\left(\chi^{2}>11.570\right)=0.021$, for the comparison with Ashkenazi Jews and Caucasian non-Jews, respectively.

\section{Chitotriosidase as a biomarker for the as-} sessment of ERT outcome

After a mean waiting time of $2.72 \pm 2.96$ years (range 0-13.5), 57 patients remaining under observation received ERT with individualised doses of recombinant glucocerebrosidase (Imiglucerase, Sanofi-Genzyme), ranging from 


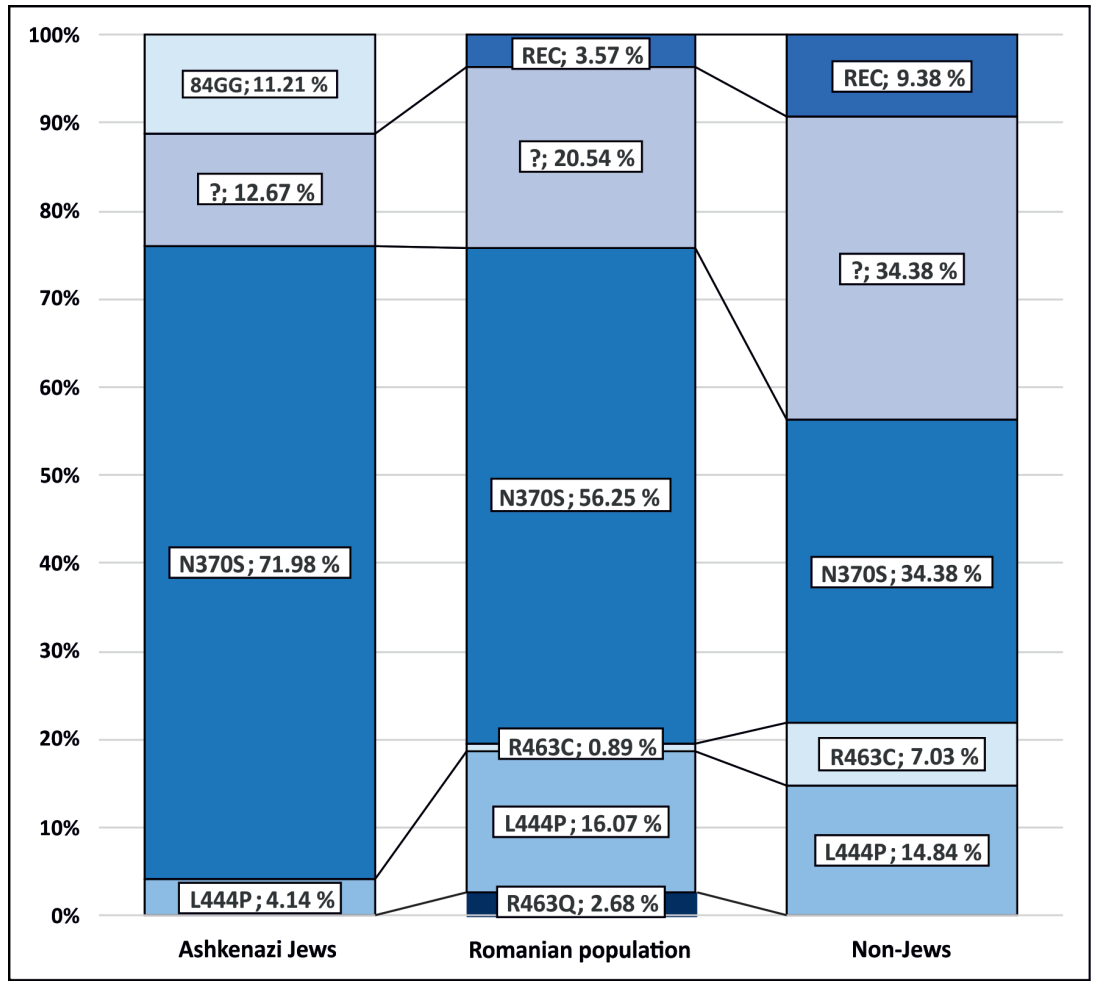

Figure 2. Allele prevalence in 56 unrelated Romanian patients with type 1 GD compared to that reported in Ashkenazi Jews and Caucasian non-Jews (18) (? designates an unknown allele and REC refers to all recombinant alleles)

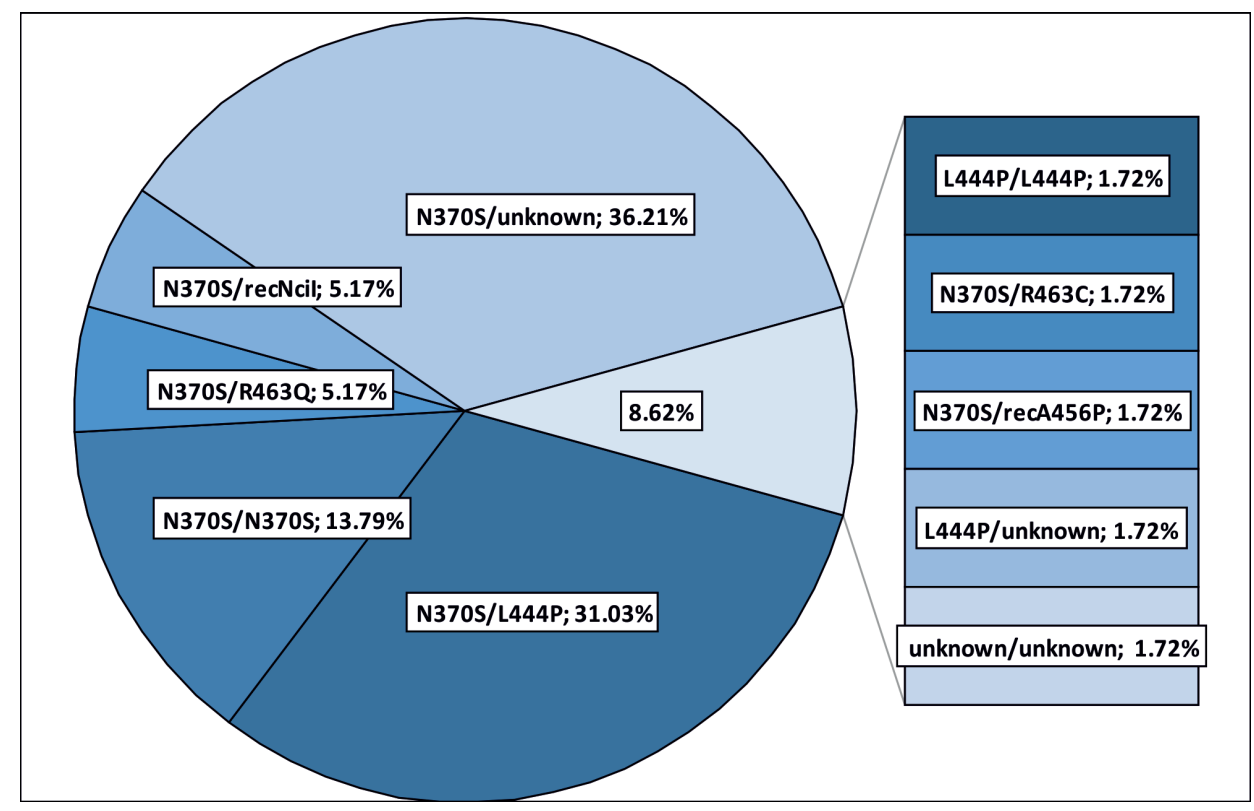

Figure 3. Genotype distribution in 58 unrelated (types 1 and 3) Romanian GD patients 


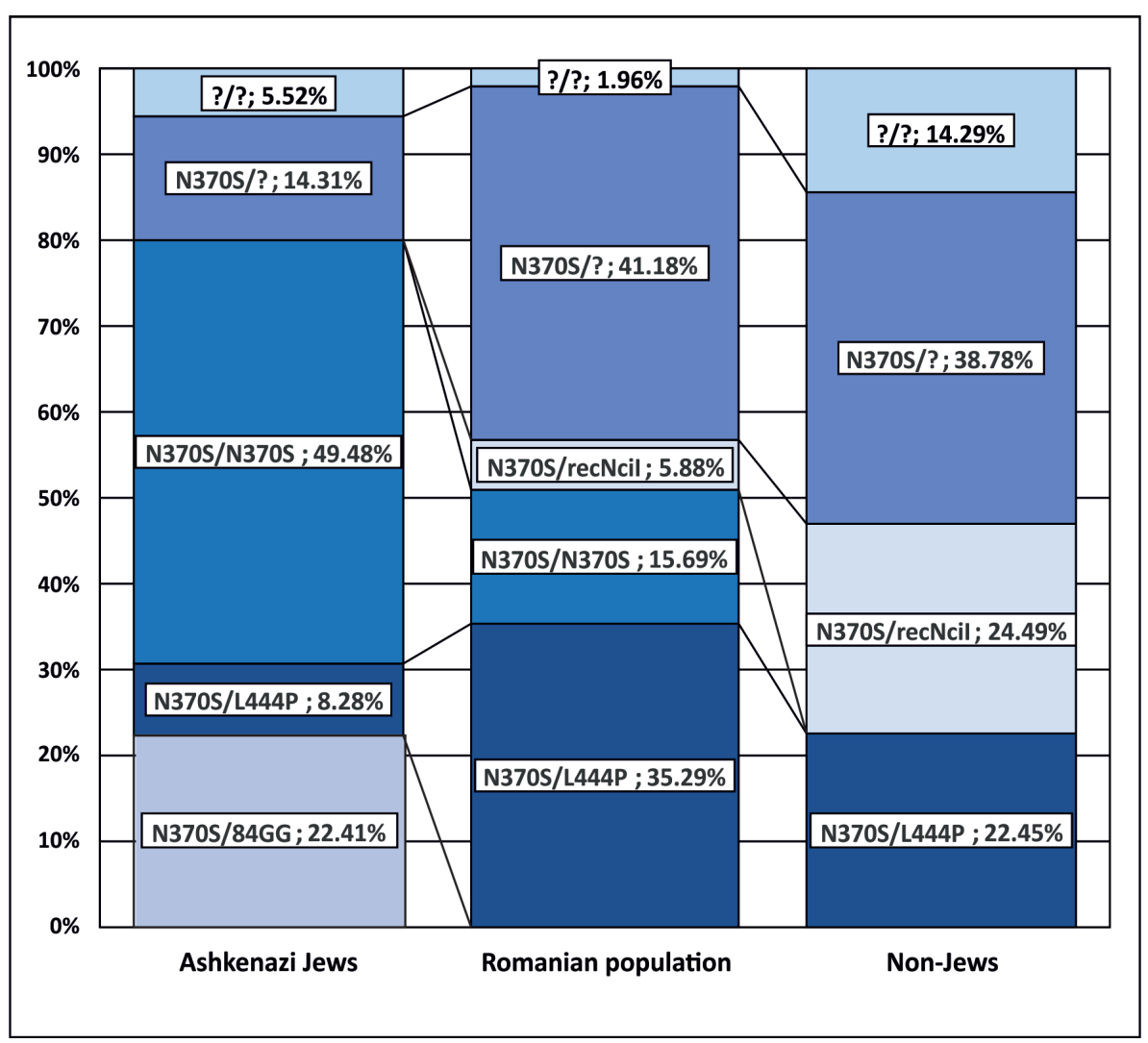

Figure 4. Genotype prevalence in 56 unrelated Romanian patients with type 1 GD compared to that reported in Ashkenazi Jews (19) and Caucasian non-Jews (18) (? designates an unknown allele)

26 to $82 \mathrm{U} / \mathrm{kg}$ body weight (mean $37.14 \mathrm{U} / \mathrm{kg}$ ), except during a supply shortage (2009-2010), which imposed lower doses (mean $25.92 \mathrm{U} / \mathrm{kg}$, range 15-50). The average duration of treatment was 5.32 years.

As a biomarker of disease evolution, chitotriosidase activity was monitored throughout the follow-up period, before and after treatment initiation. For each patient, the percentage of chitotriosidase residual activity was calculated as the ratio between the last recorded value after ERT onset and the mean pre-ERT values. Its relation to the total therapeutic dose (the sum of all bi-monthly doses, received during ERT) is illustrated in figure 5.

After the initiation of ERT, chitotriosidase residual enzymatic activity can be predicted by the following equation:
Chitotriosidase activity $(\mathrm{nmol} / \mathrm{mL} / \mathrm{h})=$ 90.45-9.93*ln (total therapeutic dose).

Based on this logarithmic regression, we found a statistically significant $(\mathrm{p}<0.001)$, moderate to good correlation between the total ERT dose and the chitotriosidase residual activity $(\mathrm{R}$ $=0.621$ ).

Chitotriosidase variation during ERT is presented in table I. In spite of numerous recordings (1533 values), its enzymatic activity (measured yearly before the start of ERT and every six months afterwards), displayed large variations. As the data were not normally distributed $(p<0.05$ for Kolmogorov-Smirnov global data analysis and Shapiro-Wilk subgroup analysis), non-parametric comparison tests were used.

Before treatment onset, no significant differences between chitotriosidase activities corre- 


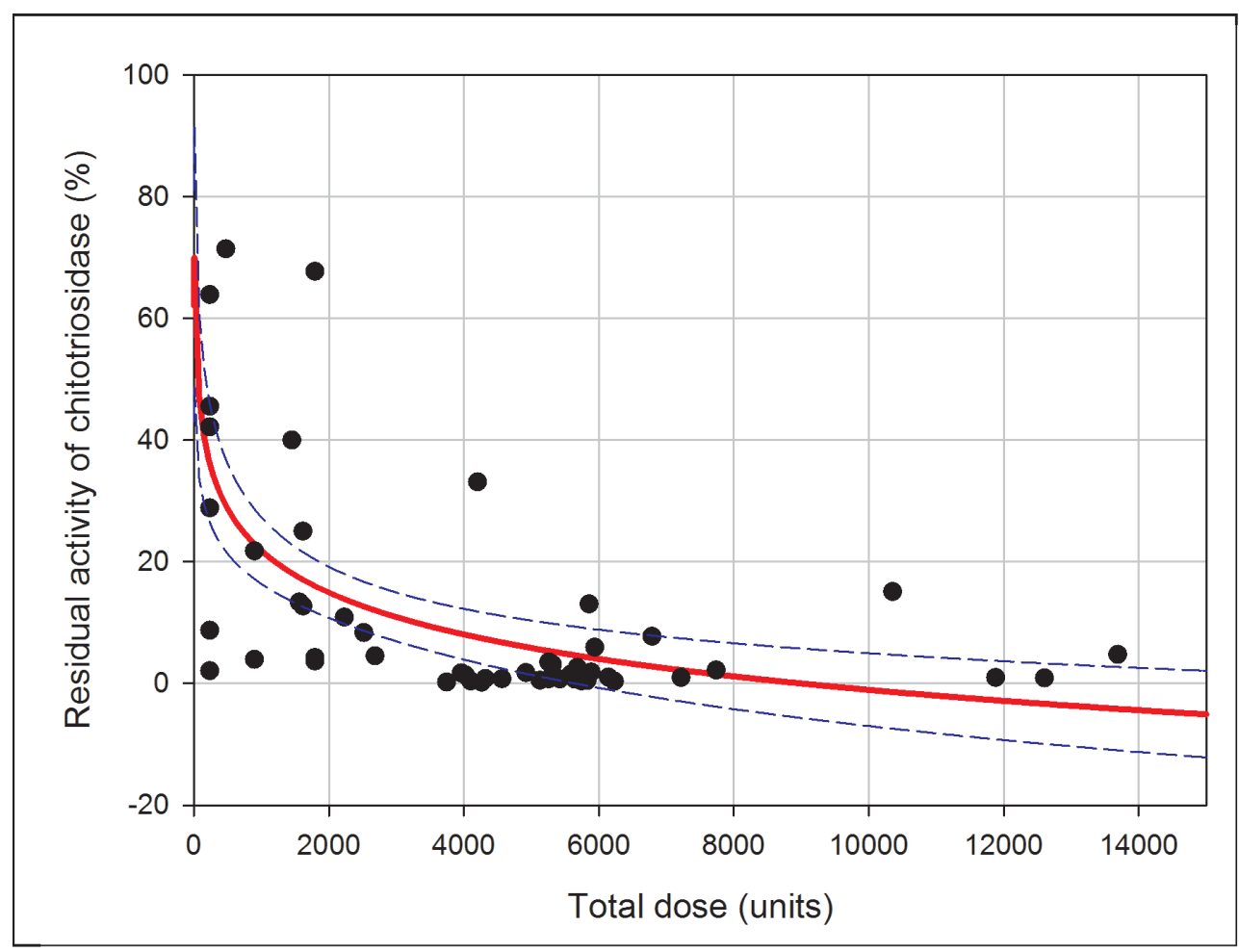

Figure 5. Relation between the total therapeutic dose and the chitotriosidase residual activity (post-ERT activity expressed as percentage of mean pre-ERT values). The red line corresponds to the predicted chitotriosidase values and the dashed lines indicate the $95 \%$ confidence interval

Table I. Evolution of 57 GD patients* under ERT

\begin{tabular}{cccccccc}
\hline Genotype & No & $\begin{array}{c}\text { Mean } \\
\text { pre-ERT } \\
\text { ChT activity }\end{array}$ & $\begin{array}{c}\text { Mean } \\
\text { waiting time } \\
\text { (years) } \\
\text { before ERT }\end{array}$ & $\begin{array}{c}\text { Mean ChT } \\
\text { activity } \\
\text { during the } \\
\text { 1st year of } \\
\text { ERT }\end{array}$ & $\begin{array}{c}\text { Mean ChT } \\
\text { activity } \\
\text { after the } \\
\text { 2nd year of } \\
\text { ERT }\end{array}$ & $\begin{array}{c}\text { Mean } \\
\text { treatment } \\
\text { time (years) }\end{array}$ & $\begin{array}{c}\text { Residual } \\
\text { ChT activ- } \\
\text { ity }\end{array}$ \\
\hline $\begin{array}{c}\text { unknown/ } \\
\text { unknown }\end{array}$ & 1 & 40500 & 0.18 & 11500 & 1449 & 5.75 & $3.58 \%$ \\
\hline N370S/R463Q & 4 & 37929 & 3.28 & 34950 & 4094 & 5.67 & $10.79 \%$ \\
\hline $\begin{array}{c}\text { N370S/ } \\
\text { unknown }\end{array}$ & 23 & 34983 & 2.86 & 21977 & 6410 & 5.51 & $18.32 \%$ \\
\hline N370S/L444P & 19 & 32767 & 2.21 & 17532 & 3434 & 4.81 & $10.48 \%$ \\
\hline N370S/N370S & 7 & 32159 & 3.91 & 29799 & 4797 & 5.23 & $14.92 \%$ \\
\hline N370S/recNciI & 1 & 23910 & 0.17 & 25500 & 21875 & 8.25 & $91.49 \%$ \\
\hline $\begin{array}{c}\text { N370S/ } \\
\text { recA456P }\end{array}$ & 1 & 16000 & 0.32 & 827 & 533 & 5.84 & $3.33 \%$ \\
\hline N370S/R463C & 1 & 13668 & 6.11 & 13000 & - & 0.33 & - \\
\hline
\end{tabular}

*Excluding the following patients: deceased (4), lost to follow-up (4) and without detectable chitotriosidase activity (4); ChT: chitotriosidase activity $(\mathrm{nmol} / \mathrm{mL} / \mathrm{h})$. Residual chitotriosidase activity: post-ERT activity expressed as percentage of mean pre-ERT values. 
sponding to the most frequent genotypes were found, indicating a similar degree of macrophage activation (Kruskal-Wallis test $\mathrm{p}=0.659$ ). These differences became apparent after two years of ERT, when we noticed statistically significant variations in chitotriosidase activity, related to the different genotypes (Kruskal-Wallis test $\mathrm{p}<0.040)$. Between each two of the 4 most frequent genotypes of treated patients (N370S/ unknown allele, N370S/L444P, N370S/N370S and N370S/R463Q), significant statistical differences were found in chitotriosidase activities, recorded after the second year of ERT (Mann-Whitney $\mathrm{U}$ tests $\mathrm{p}<0.05$ ).

\section{Discussion}

GD has been the most prevalent lysosomal storage disorder diagnosed in our laboratory during the 17 years in which this study was conducted. As a unique referral laboratory in Romania, we confirmed this diagnosis in 69 patients, most of whom had the non-neuronopathic (type 1) phenotype. There were no patients with the acute neuronopathic (type 2) GD and only 3 patients had the chronic neuronopathic (type 3 ) variant, suggesting that GD may still be underdiagnosed in our country, especially its rapidly progressive, lethal type 2 variant.

The spectrum of mutations identified in the 56 unrelated type 1 GD patients was compared to that reported in Ashkenazi Jewish and non-Jewish patients (18). After screening for the most frequent alleles, only $21 \%$ of the disease-related alleles remained unidentified in our patients, indicating an intermediate frequency of sporadic, unknown mutations, different from both reference populations. The prevalence of the N370S mutation (around 56\%) is one of the highest in Europe, comparable only to that reported in Spanish GD patients (50\%) (20). The low frequency of the L444P substitution (around 16\%) is also comparable to that of the Spanish pop- ulation $(18 \%)$, but resembles other non-Jewish East-Europeans as well $(21,22)$. A specific feature in Romanian patients is the low frequency of mutations affecting the codon 463, although a rare variant, $\mathrm{R} 463 \mathrm{Q}$, was detected besides the more common R463C substitution. Absence of the $84 \mathrm{GG}$ insertion, specific for Jewish patients (23), was not unexpected in our Caucasian, non-Jewish patients.

As a direct consequence of the high prevalence of the N370S allele, most genotypes harboured this mutation in either homozygous or heterozygous state. As expected, all these patients had type $1 \mathrm{GD}$, while patients with type 3 carried the L444P substitution, either as homozygotes, or as compound heterozygotes with an unidentified mutation. Our study further confirms these well-established genotype-phenotype correlations in GD (10). For Romanian patients with type $1 \mathrm{GD}$, the comparison of genotype frequencies with those reported in Caucasian non-Jews allowed the observation of a remarkably higher percentage of N370S/L444P compound heterozygotes (around 35\%) and N370S homozygotes $(15 \%)$. Together with the lower prevalence of N370S/recNciI genotypes and of those with two unidentified alleles, these results further differentiate our population from other non-Jewish Caucasians.

Owing to its particular geographic position, at the confluence between Eastern Europe and Asia, Romanian population may have assimilated several different ethnic groups, during its long history, but nowadays genetic homogeneity seems to prevail, as indicated by the high frequency of the N370S allele.

Circulating chitotriosidase, the most widely used biomarker for the evaluation of GD patients, was serially measured for the follow-up of our patients. Its elevated values in untreated symptomatic patients, followed by a rapid decline after the start of ERT, make it one of the most commonly used biomarker in many labo- 
ratories, including ours. The only drawback is the complete enzyme deficiency, caused by a duplication in the CHIT1 gene, which probably occurred in 4 patients investigated in this study.

Using regression analysis, we investigated the correlation between the total ERT dose received by each patient and the residual chitotriosidase activity, calculated at the end of the follow-up period. As illustrated in figure 5, chitotriosidase decline during ERT was not linear, but displayed a steep decrease after the first doses, followed by a slow, sometimes incomplete reduction. Our results are in agreement with previous reports, indicating a major decrease of chitotriosidase activity in the first months of ERT $(24,25)$. The prediction of its evolution, related to the therapeutic dose, is, in our view, a useful tool for treating physicians and therefore we propose an equation for calculating the residual chitotriosidase activity. Indeed, many authors pointed out the necessity of making quick therapeutic adjustments, based on chitotriosidase evolution, in order to prevent the occurrence of long-term complications $(24,26)$.

After the second year of ERT, we found significant differences in chitotriosidase activities corresponding to the most frequent genotypes. However, patients with the genotype N370S/ unknown allele failed to reach the normal levels of enzyme activity. This result underlines the importance of the second pathogenic allele, unidentified in this study, on disease progression under ERT. A clearly better evolution, reflected by rapid chitotriosidase decline, was observed in two patients with the genotypes N370S/recA456P and two unidentified alleles, respectively. At the opposite end of the spectrum, one patient with the genotype N370S/recNciI presented an increase in chitotriosidase levels during the first year of treatment and an almost undetectable decline afterwards, suggesting a more severe phenotype, as already reported in patients carrying the recNciI mutation (27).
Owing to the randomly scattered origin of our patients and the relatively low number of individuals originating from each geographical region of our country, our study did not identify any correlations between the geographical origin and the presence of particular mutations in the $G B A$ gene. Unlike other studies that retrospectively analysed large numbers of GD patients from many countries (28), our results do not support any conclusions about the regional distribution of the investigated mutations. Another limitation of our study is, of course, the incomplete characterisation of the spectrum of mutations in our patients. Several reports $(29,30)$ have focused on the entire sequencing of the $G B A$ gene, allowing the identification of many sporadic mutations, including novel alleles. This approach is obviously the next study direction in our laboratory.

In conclusion, our study highlights the genetic particularities of GD in Romanian patients and illustrates their specific features. In our opinion, chitotriosidase is a useful biomarker, accurately reflecting disease evolution. However, the understanding of genotype-phenotype correlations in GD is still incomplete and the prediction of disease severity cannot rely entirely on genetic factors. The identification of sporadic, yet uncharacterised, mutations in our patients is nonetheless an important step towards a better characterisation of GD.

\section{Acknowledgements}

We are grateful to the Association "Vaincre les maladies lysosomales" for supporting this study through a research project. The authors declare no conflict of interest related to the present study.
Abbreviations
GD - Gaucher disease
ERT - enzyme replacement therapy
PCR - polymerase chain reaction 


\section{References}

1. Beutler E, Grabowski GA. Gaucher disease. In: Scriver CR, Valle D, Beaudet A, Sly WS, editors. The metabolic and molecular bases of inherited disease. New York: McGraw-Hill; 2001. p. 3635-68.

2. The Human Gene Mutation Database [Internet]. Cardiff: Institute of Medical Genetics. 2006- [cited 2017 Feb 8]. Available from: http://www.hgmd.cf.ac.uk/ac/ gene.php?gene $=\mathrm{GBA}$.

3. Hughes DA, Pastores GM. The pathophysiology of GD - current understanding and rationale for existing and emerging therapeutic approaches. Wien Med Wochenschr. 2010 Dec;160(23-24):594-9. DOI: 10.1007/ s10354-010-0864-4

4. Mistry PK, Lopez G, Schiffmann R, Barton NW, Weinreb NJ, Sidransky E. Gaucher disease: Progress and ongoing challenges. Mol Genet Metab. 2017 Jan-Feb;120(1-2):8-21. DOI: 10.1016/j. ymgme.2016.11.006

5. Aerts JM, Hollak CE. Plasma and metabolic abnormalities in Gaucher's disease. Bailliere's Clin Haematol. 1997 Dec;10(4):691-709. DOI: 10.1016/S09503536(97)80034-0

6. van Dussen L, Hendriks JE, Groener JEM, Boot RG, Hollak CE, Aerts JM. Value of plasma chitotriosidase to assess non-neuronopathic Gaucher disease severity and progression in the era of enzyme replacement therapy. J Inherit Metab Dis. 2014 Nov;37(6):991-1001. DOI: 10.1007/s10545-014-9711-x

7. Jeong SY, Park SJ, Kim HJ. Clinical and genetic characteristics of Korean patients with Gaucher disease. Blood Cells Mol Dis. 2011 Jan;46(1):11-4. DOI: 10.1016/j.bcmd.2010.07.010

8. Karaca E, Kalkan S, Onay H, Aykut A, Coker M, Ozkinay F. Analysis of the $\beta$-glucocerebrosidase gene in Turkish Gaucher disease patients: mutation profile and description of a novel mutant allele. J Pediatr Endocrinol Metab. 2012;25(9-10):957-62. DOI: 10.1515/jpem2012-0155

9. Hruska KS, LaMarca ME, Scott CR, Sidransky E. Gaucher disease: mutation and polymorphism spectrum in the glucocerebrosidase gene (GBA). Hum Mutat. 2008 May;29(5):567-83. DOI: 10.1002/humu.20676

10. Hruska KS, LaMarca ME, Sidransky E. Gaucher disease: molecular biology and genotype-phenotype correlations. In: Futerman AH, Zimran A, editors. Gaucher disease. Boca Raton (FL): CRC Press; 2007. p. 13-48.

11. Drugan C, Procopciuc L, Jebeleanu G, Grigorescu-Sido P, Dussau J, Poenaru L, et al. Gaucher disease in Romanian patients: incidence of the most common mutations and phenotypic manifestations. Eur J Hum Genet. 2002 Sep;10(9):511-5. DOI: 10.1038/sj.ejhg.5200845

12. Peters SP, Coyle P, Glew RH. Differenciation of beta-glucocerebrosidase from beta-glucosidase in human tissues using sodium taurocholate. Arch Biochem Biophys. 1976 Aug;175(2):569-82. DOI: 10.1016/00039861(76)90547-6

13. Beutler E, Gelbart T, West C. The facile detection of the nt 1226 mutation of glucocerebrosidase by "mismatched" PCR. Clin Chim Acta. 1990 Dec;194(23):161-6. DOI: 10.1016/0009-8981(90)90130-K

14. Dahl N, Lagerström M, Erikson A, Pettersson U. Simplified detection of Nci mutation in Gaucher disease. Lancet. 1990 Jun;335(8705):1589-90. DOI: 10.1016/0140-6736(90)91414-6

15. Beutler E, Gelbart T, Kuhl W, Sorge J, West C. Identification of the second common Jewish Gaucher disease mutation makes possible population-based screening for the heterozygous state. Proc Natl Acad Sci USA. 1991 Dec;88(23):10544-7. DOI: 10.1073/ pnas.88.23.10544

16. Hollak CE, van Weely S, van Oers MH, Aerts JM. Marked elevation of plasma chitotriosidase activity. A novel hallmark of Gaucher disease. J Clin Invest. 1994 Mar;93(3):1288-92. DOI: 10.1172/JCI117084

17. Boot RG, Renkema GH, Verhoek M, Strijland A, Bliek J, de Meulemeester TM, et al. The human chitotriosidase gene. Nature of inherited enzyme deficiency. J Biol Chem. 1998 Oct;273(40):25680-5. DOI: 10.1074/ jbc. 273.40 .25680

18. Koprivica V, Stone DL, Park JK, Callahan M, Frisch A, Cohen IJ, et al. Analysis and classification of $304 \mathrm{mu}-$ tant alleles in patients with type 1 and type 3 Gaucher disease. Am J Hum Genet. 2000 Jun; 66(6):1777-86. DOI: $10.1086 / 302925$

19. Grabowski GA, Horowitz M. Gaucher's disease: molecular, genetic and enzymological aspects. Bailliere's Clin Haematol. 1997 Dec;10(4):635-56. DOI: 10.1016/ S0950-3536(97)80032-7

20. Alfonso P, Aznarez S, Giralt M, Pocovi M, Giraldo P. Mutation analysis and genotype/phenotype relationships of Gaucher disease patients in Spain. J Hum Genet. 2007 Apr;52(5):391-6. DOI: 10.1007/s10038-007- 
0135-4

21. Mattošová S, Chandoga J, Hlavatá A, Saligová J, Maceková D. Spectrum of GBA mutations in patients with Gaucher disease from Slovakia: identification of five novel mutations. Isr Med Assoc J. 2015 Mar;17(3):16670.

22. Erdos M, Hodanova K, Taskó S, Palicz A, Stolnaja L, Dvorakova L, et al. Genetic and clinical features of patients with Gaucher disease in Hungary. Blood Cells Mol Dis. 2007 Jul-Aug;39(1):119-23. DOI: 10.1016/j. bcmd.2007.02.005

23. Horowitz M, Zimran A. Mutations causing Gaucher disease. Hum Mutat. 1994;3(1):1-11. DOI: 10.1002/ humu. 1380030102

24. Cabrera-Salazar MA, O'Rourke E, Henderson N, Wessel H, Barranger JA. Correlation of surrogate markers of Gaucher disease. Implications for long-term follow up of enzyme replacement therapy. Clin Chim Acta. 2004 Jun;344(1-2):101-7. DOI: 10.1016/j.cccn.2004.02.018

25. Drugan C, Drugan TC, Grigorescu-Sido P, Naşcu I. Modelling long-term evolution of chitotriosidase in non-neuronopathic Gaucher disease. Scand J Clin Lab Invest. 2017;77(4):275-82. DOI: 10.1080/00365513.2017.1303191

26. Poll LW, Koch JA, Willers R, Aerts H, Scherer A,
Häussinger D, et al. Correlation of bone marrow response with hematological, biochemical, and visceral responses to enzyme replacement therapy of nonneuronopathic (type 1) Gaucher disease in 30 adult patients. Blood Cells Mol Dis. 2002 Mar-Apr;28(2):209-20. DOI: $10.1006 / \mathrm{bcmd} .2002 .0511$

27. Choy FY, Zhang W, Shi HP, Zay A, Campbell T, Tang $\mathrm{N}$, et al. Gaucher disease among Chinese patients: review on genotype/phenotype correlation from 29 patients and identification of novel and rare alleles. Blood Cells Mol Dis. 2007 May-Jun;38(3):287-93. DOI: 10.1016/j.bcmd.2006.11.003

28. Bronstein S, Karpati M, Peleg L. An update of Gaucher mutations distribution in the Ashkenazi Jewish population: prevalence and country of origin of the mutation R496H. Isr Med Assoc J. 2014 Nov;16(11):683-5.

29. Malini E, Grossi S, Deganuto M, Rosano C, Parini R, Dominisini S, et al. Functional analysis of 11 novel GBA alleles. Eur J Hum Genet. 2014 Apr;22(4):511-6. DOI: 10.1038/ejhg.2013.182

30. Ankleshwaria C, Mistri M, Bavdekar A, Muranjan M, Dave U, Tamhankar P, et al. Novel mutations in the glucocerebrosidase gene of Indian patients with Gaucher disease. J Hum Genet. 2014 Apr;59(4):223-8. DOI: 10.1038/jhg.2014.5 